\title{
La duración en oraciones sin expansión en la voz femenina de dos países fronterizos: Colombia (Bogotá-Medellín) y Venezuela (Caracas-Mérida)*
}

\author{
Mercedes Amparo Muñetón Ayala** \\ Chaxiraxi Díaz Cabrera** \\ Josefa Dorta Luis ${ }^{* * * *}$
}

\section{Resumen}

Este trabajo se realizó en el marco del proyecto AMPER. Se comparó la duración entre oraciones declarativas e interrogativas de voces femeninas de dos países fronterizos: Colombia y Venezuela. Se analizó la media de las oraciones en función de la tipología acentual, las diferencias mínimas perceptibles y, finalmente, se generó la estructura de la duración. Los resultados muestran que las tónicas tienden a ser significativamente más largas que las vocales adyacentes. Pero, Colombia a diferencia de Venezuela, presenta una mayor duración y su estructura difiere entre las modalidades.

Palabras clave: Prosodia, duración, comparación interlingüística, diferencias mínimas perceptibles.

\section{The duration in sentences without expansion in the female voice of two bordering countries: Colombia (Bogotá- Medellín) and Venezuela (Caracas-Mérida)}

\begin{abstract}
This research has been conducted as part of the framework of the international AMPER. The objective was to compare the duration between declarative and interrogative sentences of female voices from two border areas: Colombia and Venezuela. In order to do so, the following was analyzed: the average of the sentences taking into account the stress of the words; the just noticeable differences, and the duration structure. The results reveal a tendency of a general alignment between the larger duration with tonic vowels. However, Colombia
\end{abstract}

\footnotetext{
* El trabajo se vincula a dos proyectos de investigación: Relaciones entre el límite prosódico y el límite sintagmático en oraciones declarativas e interrogativas de voces femeninas y masculinas de Colombia e Islas Canarias (España), UdeA, CODI, IP Mercedes Muñetón Ayala; y Estudio comparativo de la entonación y del acento en zonas fronterizas del español (FFI2014-52716-P), proyecto de I+D del Programa Estatal de Fomento de la Investigación Científica y Técnica de Excelencia, MINECO de España, IP Josefa Dorta.

** Colombiana, Dra. en Psicolingüística experimental y aplicada, profesora vinculada de la Universidad de Antioquia, Colombia. mercedes.muneton@udea.edu.co

*** Española, Dra. en Lingüística general, investigadora del GI ProFonDis de la Universidad de La Laguna, España.chadiaz@ull.edu.es

**** Española, Dra. en Filología hispánica, Catedrática de Lingüística General y profesora de la Universidad de La Laguna, España. jdorta@ull.edu.es
} 
differs from Venezuela because it presents larger duration and the structure differs between modalities.

Key words: Prosody, Duration, Crosslinguistic comparison, Just-noticeable difference.

\section{Introducción}

Independientemente del sistema lingüístico, la prosodia es un determinante intrínseco de la lengua hablada (Cutler, Dalan y Van Doselaar, 1997) conformada por parámetros acústicos como la frecuencia fundamental, la intensidad y la duración. Cada uno de estos parámetros modifica el contorno melódico de una manera específica hasta el punto de que permite diferenciar la procedencia de hablantes de un mismo sistema lingüístico. Por ejemplo, la lengua oficial de Colombia y Venezuela es el español; estos dos países comparten frontera y, sin embargo, las características prosódicas permiten identificar el origen de sus hablantes.

La duración, parámetro de estudio en el presente trabajo, se refiere a la prolongación temporal de un segmento de habla (Fox, 2000). Este parámetro prosódico, denominado también cantidad, es el correlato acústico del tiempo. Al respecto, hay que tener en cuenta que cada segmento lingüístico para ser percibido debe tener una duración mínima que oscila entre 10 y 40 ms; no obstante, como anota Marrero (2008), la mayor sensibilidad natural emerge en el rango que va de 40 a $60 \mathrm{~ms}$. La importancia de la duración ha sido destacada por numerosos autores; citemos, por ejemplo, a Andrés Bello (1955), para quien el tiempo es importante en la producción silábica con el fin de alcanzar el ritmo. Otros autores se han referido a su importancia en la interpretación léxica ${ }^{1}$ (Salverda, Dahan y McQueen, 2003) o en la relación entre el contenido semántico y el procesamiento métrico en francés (Cyrille y otros, 2007)².

Para ello, grabaron los movimientos oculares de participantes holandeses mientras escuchaban oraciones y veían cuatro dibujos de objetos en la pantalla del ordenador. A los participantes se les instruyó para pulsar sobre el objeto mencionado en la oración. Los ítems fueron interpretados como palabras monosilábicas (ham) más que como bisilábicas (hámster) cuando la duración de la primera sílaba fue más larga que cuando fue más corta.

2 El estudio se llevó a cabo bajo dos condiciones: una semántica y una métrica. Los participantes escucharon oraciones que finalizaban con palabras trisílabas que eran congruentes o incongruentes desde un punto de vista semántico o desde un punto de vista métrico. Los datos mostraron que se juzgó 
Centrándonos en el español, en diversos estudios se ha analizado la duración en relación con el ritmo, el acento o, más recientemente, la entonación, aunque no siempre se le da la misma relevancia (Canellada y Madsen, 1987; Dorta y Mora, 2011; Fernández Planas y Martínez Celdrán, 2003; Garrido, Llisterri, Mota y Ríos, 1995; Llisterri, Machuca, de la Mota, Riera y Ríos, 2005; Toledo, 2000). No obstante, en lo que coinciden la mayoría de los investigadores es que las sílabas acentuadas presentan una mayor duración que las no acentuadas (Alfano, Saby y Llisterri, 2007; Canellada y Madsen, 1987), aunque no siempre las diferencias de duración superan los diferentes umbrales perceptivos considerados (Dorta, Hernández y Díaz, 2011; Dorta y Jorge Trujillo, 2015; Pamies Bertrán y Fernández Planas, 2006; Muñetón, 2016).

Uno de los principales objetivos del proyecto AMPER-internacional ${ }^{3}$ es contrastar diferentes lenguas románicas y sus variedades o dialectos con el fin de determinar las características prosódicas específicas de las mismas. De esta forma, el presente trabajo colabora en el desarrollo del actual objetivo comparando dos variedades del español, esto es, el colombiano con el venezolano. En la mayoría de los trabajos vinculados a este proyecto, como en otros, se ha priorizado el estudio de la Fo sobre el de duración e intensidad (Dorta, Hernández y Díaz, 2009; Escandell, 2011). Concretamente, en lo que se refiere a la comparación entre Venezuela y Colombia, citamos dos trabajos recientes en los que el objetivo de estudio es la comparación tonal: Díaz, Dorta, Mora y Muñetón (en prensa) compararon la frecuencia fundamental en voces femeninas de dos de las ciudades más importantes ubicadas en la cordillera de los Andes, esto es, Mérida (capital del estado de Mérida, en Venezuela) y Medellín (capital del Departamento de Antioquia, en Colombia). Los principales resultados revelaron que el patrón nuclear predominante en ambos países era el circunflejo. Sin embargo, otro estudio de tipo comparativo (Díaz, Muñetón y Dorta, 2017), centrado en las capitales de ambos países, esto es, Bogotá (Colombia) y Caracas (Venezuela) mostró como resultado principal que las dos zonas de estudio presentan configuraciones tonales diferentes en las oraciones interrogativas.

la incongruencia métrica como incongruencia semántica, aun cuando se les había pedido que atendieran solo a la parte semántica.

3 Los coordinadores generales hasta el mediados de 2015 han sido Michel Contini (Université Stendhal-Grenoble 3) y Antonio Romano (Università di Torino). Este último es el coordinador general en la actualidad. 
Por lo que respecta a la comparación entre Colombia y Venezuela basada en el análisis de la duración, podemos mencionar el trabajo de Dorta y Mora (2011) en el que la duración se estudia en relación con el ritmo. El análisis se centró en emisiones de mujeres canarias de la isla de la Palma (Islas Canarias) y de mujeres de Mérida (Venezuela). Los resultados niegan el isosilabismo en las localidades analizadas. Otros resultados similares para el español de Venezuela se pueden ver en Mora y Asuaje (2009) y en Mora, Villamizar, Blondet y López (1999), mientras que para algunas Islas Canarias se ha sugerido una tendencia al isosilabismo (Almeida, 1999). Para Colombia podemos mencionar un trabajo reciente realizado por Muñetón (2016) en el cual, aunque se estudian los tres parámetros acústicos en voz masculina de Medellín, los resultados principales relacionados con la duración mostraron que independientemente de la estructura sintáctica ${ }^{4}$ y de la tipología acentual ${ }^{5}$, cuando hubo DMP (diferencias mínimas perceptibles) la vocal tónica fue siempre la más larga. Además, la Fo y la duración correlacionaron de forma negativa en las vocales tónicas. Por consiguiente, a mayor duración menor Fo en las vocales acentuadas.

\section{Objetivo y variables}

El objetivo del presente estudio es comparar la duración en el habla de informantes femeninas de ciudades muy importantes de dos países fronterizos. De cada país se eligió la capital y una de las ciudades más importantes ubicadas en la cordillera de Los Andes: en Venezuela, Caracas y Mérida; en Colombia, Bogotá y Medellín.

Analizamos, en primer lugar, la duración vocálica con el objeto de obtener una media de las mismas, luego las DMP y, finalmente, se generó la estructura de la duración basándonos en una propuesta que se verá a continuación de largas y breves.

Las variables que se tendrán en cuenta en el presente estudio son: 1. Variedad del español: venezolano vs. colombiano; 2. Modalidad: declarativa vs. interrogativa; 3. Tipología oracional: se refiere a las oraciones que se generan al combinar el SN (O-P-Pro) con el SP (O-P-Pro) $; 4$.

La estructura sintáctica se refiere a las oraciones sin expansión: el saxofón se toca con emoción, con expansión en el sujeto: la máquina clásica se toca con pánico; o con expansión en el objeto: la máquina se toca con pánico práctico.

Tipología acentual: palabras oxítonas, paroxítonas y proparoxítonas.

SN: sintagma nominal; SP: sintagma preposicional; O: oxítona; P: paroxítona; Pro: proparoxítona 
Sintagmas: nominal vs. verbal vs. preposicional; 5. Tipología acentual: oxítona vs. paroxítona vs. proparoxítona; 6. Acento: tónico vs. átona. El estudio se justifica en primer lugar, por la escasez de trabajos relacionados con el tema de análisis y, en segundo lugar, porque intenta mostrar el comportamiento sistemático de la duración en dos países vecinos que comparten la misma lengua.

\section{Método y procedimiento}

\subsection{Propuesta de configuraciones durativas}

Una de las etiquetas más utilizadas tradicionalmente para marcar las diferencias de duración vocálicas o silábicas es larga vs. breve. Sin embargo, este etiquetaje no deja ver la relación simultánea entre la pretónica, tónica y postónica. Con el propósito de dar cuenta de esta triple relación, Fernández Planas y Martínez Celdrán (2003) hacen una propuesta que emula los principios del etiquetaje de Fo del Modelo Métrico Autosegmental partiendo de dos dimensiones durativas G (grande) y P (pequeña). Nuestro propósito es, igualmente, contrastar simultáneamente la tónica con sus adyacentes átonas, pero identificando las vocales como largas (L) y breves (B) puesto que consideramos que estas etiquetas, tradicionalmente utilizadas, son más adecuadas para hacer referencia a las diferencias de duración. Además, no emulamos el Modelo Métrico Autosegmental para generar las configuraciones de duración, sino que extraemos las estructuras de duración que subyacen a la vocal tónica en relación con sus adyacentes.

Nuestra propuesta de configuraciones de duración es abierta por lo que ha de irse revisando y completando en sucesivos trabajos donde se analicen corpus más amplios y espontáneos. En la propuesta subyace el concepto de diferencias mínimas perceptibles (DMP), debido a que acota diferencias durativas funcionales a nivel cognitivo. Además, la relativización de los datos permite eliminar diferencias en dos niveles: intrasujeto e intersujeto. Los datos relativos nos dan una medida objetiva para determinar patrones entre hablantes o tipologías oracionales diferentes. Con el fin de poder diferenciar la tónica de las vocales adyacentes, independientemente de que sea larga o breve, la marcaremos con un asterisco. La definición y posición de cada estructura en nuestra propuesta es la siguiente: 1) L*: la tónica es significativamente más larga 
que la pretónica y postónica. 2) B*: no hay diferencias significativas entre la pretónica-tónica, ni entre tónica-postónica. 3) BL*: solo hay diferencias entre pretónica-tónica, siendo la tónica la más larga. 4) L*B: solo hay diferencias entre tónica-postónica, siendo la tónica la más larga. 5) LB*: solo hay diferencias entre la pretónica-tónica, siendo la pretónica la más larga. 6) B*L: solo hay diferencias entre tónica-postónica, siendo la postónica la más larga. 7) BL*L: la tónica es más larga que la pretónica y a su vez la postónica es más larga que la tónica.

\subsection{Informantes y corpus}

Se analiza la duración en emisiones de cuatro informantes femeninas sin estudios, cada una perteneciente a la zona urbana de un punto de encuesta: Medellín y Bogotá (Colombia) y Mérida y Caracas (Venezuela). En cada punto urbano se entrevistó a una mujer con edad comprendida en el rango de 25-45 años. El material utilizado se compone de 216 oraciones sin expansión (108 declarativas y otras tantas interrogativas) tomadas del corpus fijo del proyecto AMPER-Col y AMPER-Ven. La estructura del corpus es del tipo SVO (sujeto+verbo+objeto), en el cual los sintagmas nominal y preposicional están compuestos por trisílabos de diferente tipología acentual (oxítona, paroxítona, proparoxítona); el sintagma verbal permanece invariable (acento paroxítono). Las oraciones son del tipo $E l$ saxofón se toca con emoción; La guitarra se toca con paciencia, la máquina se toca con pánico y todas las combinaciones acentuales posibles en las fronteras inicial y final. El estudio de los datos se hizo a partir de la media de las 3 repeticiones de cada oración.

\subsection{Análisis acústico}

Utilizamos el programa MatLab -Matriz Laboratory- ${ }^{7}$ para el análisis acústico ${ }^{8}$. Para la relativización de la duración usamos el umbral diferencial perceptivo de 33.33\% (Fernández Planas y Martínez Celdrán, 2003). Con el objetivo de identificar las DMP, los datos se organizan en dos tipos de contrastes: pretónica-tónica y tónica-postónica. El procedimiento para

Licencia no 256105 del Laboratorio de Fonética de la Universidad de La Laguna (España) y Licencia n878004 del laboratorio de fonética de la Universidad de Antioquia (Colombia). Para más información sobre el programa y las subrutinas creadas por el Centre de Dialectologie de Grenoble, véase Fernández Planas (2005); sobre la adaptación realizada por el grupo Amper-Astur, véase López Bobo y otros, 2007. 8 La segmentación de las vocales fue realizada por dos investigadores que recibieron entrenamiento en el marco del grupo de investigación PROFONDIS, coordinado por la profesora Josefa Dorta Luis. 
generar el umbral es como sigue: 1) en cada contraste la vocal más larga corresponde al $100 \%$; 2 ) se hace una regla de tres y se obtiene el porcentaje de la vocal más corta; 3 ) se hace una resta entre el 100\% y el porcentaje de la vocal corta con el fin de obtener el valor relativo; 4) si este valor es igual o mayor a $33.33 \%$ se considera que hay DMP entre las dos vocales. En el análisis de los datos con DMP y de la estructura de la duración se usó la prueba estadística no paramétrica de Chi al cuadrado con el fin de generar unos resultados lo más objetivos posibles de la distribución de la muestra. Esta prueba compara la frecuencia de los datos observados con la frecuencia de los esperados. Así, el estadístico Chi-cuadrado pondera qué tanto varía la distribución observada de conteos con respecto a la distribución hipotética. Si la distribución de las variables comparadas es igual entonces no se encontrarán diferencias entre ellas, pero si es diferente sí las hay. El valor significativo se sitúa en 0.05 .

\section{Resultados}

Presentaremos los resultados en tres grandes apartados: en el primero (4.1) veremos el comportamiento de la duración basado en el análisis de las medias generales en función de la tipología acentual; en el segundo (4.2) observaremos la distribución de las DMP, y en el tercero (4.3) la propuesta de la estructura de la duración.

\subsection{Análisis de las medias generales}

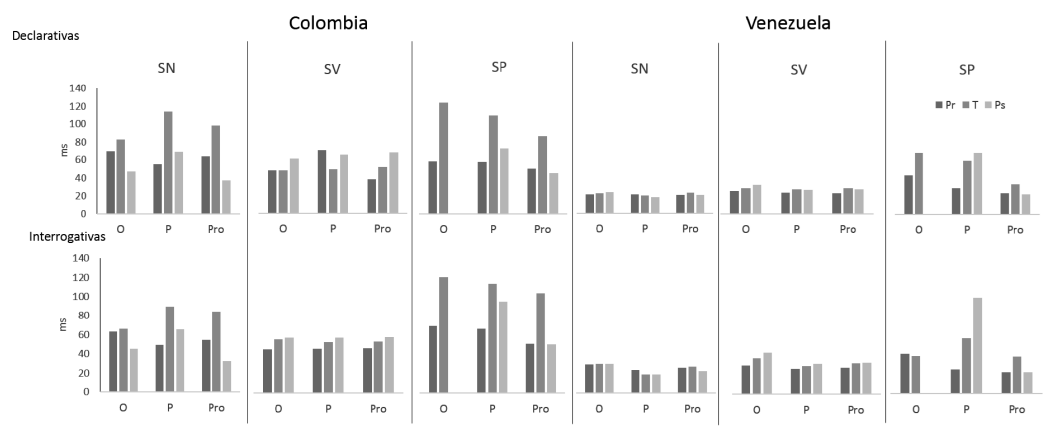

Figura 1. Oraciones declarativas e interrogativas en función de los sintagmas y la tipología acentual ${ }^{9}$.

$9 \quad$ Las abreviaturas de la figura son: SN: sintagma nominal; SV: sintagma verbal; SP: SP: sintagma preposicional; O: oxítona; P: paroxítona; Pro: proparoxítona; Pr: pretónica, T: tónica, Ps: Postónica. 
Tomando las oraciones en conjunto, la figura 1, muestra que la duración de las vocales es mayor en Colombia que en Venezuela. Observamos que en Colombia la tónica del SN y del SP presenta mayor duración que la vocal precedente y siguiente, independientemente de la modalidad y de la tipología acentual. En cambio, en el SV de las tres tipologías acentuales dela modalidad interrogativa la duración es creciente, esto es, la más corta es la Pr y las más larga es la Ps. En las declarativas se presenta este mismo patrón, pero solo en las proparoxítonas; en las paroxítonas, la Pr y la Ps son más largas que la tónica, y en las oxítonas la tónica y la Pr son más cortas que la Ps. En Venezuela, por su parte, el SN de ambas modalidades tienden a presentar duraciones constantes independientemente de la tipología acentual: en el SV la duración es ascendente en las oxítonas siendo la más corta la Pr, y la más larga la Ps; en el SP las postónicas de las paroxítonas tienden a ser más largas que las tónicas; también las tónicas de las proparoxítonas tienden a ser más largas que la pretónica y la tónica. Sin embargo, la tónica de la oxítona en la modalidad declarativa es más larga que la pretónica.

En resumen, en ambos países, la tónica en el SP presenta mayor duración en las oxítonasy menor duración en las proparoxítonas. No obstante, este patrón no se aplica para el SP de las interrogativas de Venezuela, en donde las paroxítonas tienen la mayor duración y las proparoxítonas la menor. Sin embargo, en ningún caso las proparoxítonas son las más largas. Esto indica que la duración es directamente proporcional al lugar del acento en la oración, es decir, cuanto más tardía esté la tónica en el SP más larga es la vocal (véase la tabla 1). 
Tabla 1. Medias vocálicas en las oraciones declarativas e interrogativas en función de los sintagmas y la tipología acentual ${ }^{10}$.

\begin{tabular}{|c|c|c|c|c|c|c|c|c|}
\hline & \multicolumn{3}{|c|}{ Colombia } & \multirow[b]{2}{*}{ Pro } & \multicolumn{3}{|c|}{ Venezuela } \\
\hline & & & 0 & $\mathbf{P}$ & & 0 & $\mathbf{P}$ & Pro \\
\hline \multirow{9}{*}{ Declarativas } & \multirow{3}{*}{ SN } & Pr & 70 & 55 & 64 & 23 & 24 & 23 \\
\hline & & $\mathbf{T}$ & 83 & 114 & 99 & 25 & 22 & 25 \\
\hline & & Ps & 47 & 69 & 37 & 26 & 20 & 23 \\
\hline & \multirow{3}{*}{ SV } & Pr & 47 & 48 & 51 & 26 & 24 & 23 \\
\hline & & $\mathbf{T}$ & 60 & 64 & 66 & 29 & 28 & 29 \\
\hline & & Ps & 61 & 61 & 58 & 33 & 27 & 28 \\
\hline & \multirow{3}{*}{ SP } & Pr & 65 & 64 & 56 & 43 & 28 & 23 \\
\hline & & $\mathbf{T}$ & 137 & 122 & 96 & 68 & 59 & 34 \\
\hline & & Ps & -- & 81 & 51 & -- & 68 & 22 \\
\hline & & & & & & & & \\
\hline \multirow{9}{*}{ Interrogativas } & \multirow{3}{*}{ SN } & Pr & 64 & 50 & 55 & 25 & 20 & 22 \\
\hline & & $\mathbf{T}$ & 66 & 89 & 84 & 25 & 16 & 23 \\
\hline & & Ps & 46 & 66 & 33 & 25 & 16 & 19 \\
\hline & \multirow{3}{*}{ SV } & Pr & 46 & 46 & 47 & 25 & 22 & 23 \\
\hline & & $\mathbf{T}$ & 57 & 54 & 54 & 32 & 25 & 27 \\
\hline & & Ps & 58 & 58 & 59 & 36 & 27 & 28 \\
\hline & \multirow{3}{*}{ SP } & Pr & 65 & 63 & 48 & 42 & 26 & 23 \\
\hline & & $\mathbf{T}$ & 113 & 106 & 97 & 40 & 59 & 39 \\
\hline & & Ps & -- & 89 & 48 & -- & 102 & 23 \\
\hline
\end{tabular}

Por otra parte, al comparar las medias de las tónicas del SN con el SP se observa que las de este último son más largas. Si hacemos la misma comparación, pero teniendo en cuenta las DMP, encontramos que independientemente de la modalidad la tónica del SP suele ser perceptivamente más larga. Así, hay DMP en Colombia en las oxítonas en ambas modalidades, y en Venezuela en las tres tipologías acentuales, exceptuando las proparoxítonas de las declarativas, en las que no hay DMP.

\subsection{Distribución de las diferencias mínimas perceptivas (DMP)}

\subsubsection{Datos generales}

El número de vocales tónicas y pretónicas de toda la muestra es idéntico (216 en cada caso); el de postónicas es de 192. La comparación de la Pr con la T y la T con la Ps genera en total 408 contrastes, la mitad corresponde a las declarativas y la otra mitad a las interrogativas. De esta com-

10 Las abreviaturas de la tabla son: SV: sintagma verbal; Pr: pretónica, T: tónica, Ps: Postónica, O: oxítona, P: paroxítona y Pro: proparoxítona. 
paración los datos absolutos (valores directos) muestran que la tónica es larga en relación a la pretónica o postónicaen el $76.47 \%$ y esbreve en relación a la pretónica y postónica en un $8.33 \%$ y un $15.20 \%$ de los casos, respectivamente. En lo que respecta a los datos relativos, del 100\% (138 casos) de contrastes con DMP encontramos que enel $92.03 \%$ la mayor duración recae en las tónicas, el 4.34\% en las pretónicas y el 3.62\% en las postónicas. Solo en el $\mathbf{2 5 . 1 9 \%}$ de los casos la tónica presenta diferencias simultáneamente con la pretónica y postónica.

Tomando las oraciones en conjunto, los valores relativos muestran (tabla 2 y 3 ) que existen diferencias significativas en función de las localidades $\chi^{2}(3)=23.73, p=.000$, siendo Medellín (con un 39.87\%) la que muestra un porcentaje mayor de DMP, luego Bogotá (26.81\%), a continuación, Caracas (22.46\%) y, finalmente, Mérida (10.86\%).

En Colombia, no hay diferencia entre las modalidades $\chi^{2}=1.56, \mathrm{p}=.211$, ni entre la tipología acentual oracional $\chi^{2}(8)=7.00, p=.537$, pero sí en la distribución de las DMP en función de los sintagmas $\chi^{2}(2)=30.02, p=$ .ooo, siendo el SV (6.52\%) el que menos DMP presenta en comparación con el SN (44.56\%) y el SP (48.92\%). La mayor duración con DMP recae en las tónicas. En el contaste entre Pr-T y la T-Ps $\chi^{2}=4.34$, p = .o37; siendo el primer contraste el que presenta una distribución mayor (60.87\% y 39.13\% respectivamente). En el contraste tipología de palabras $\chi^{2}(2)=10.58, \mathrm{p}=$ .005, la oxítona (17.39\%) es la que menos presenta DMP en comparación con las proparoxítonas (42.39\%) y las paroxítonas (40.21\%). En Venezuela, no hay diferencia entre las modalidades $\chi^{2}=0.78, p=.376$, la tipología acentual oracional $\chi^{2}(8)=5.52, \mathrm{p}=.961$ y el contraste entre las tipologías de las palabras $\chi^{2}(2)=4.21, p=.121$, pero, como en Colombia, sí hay en la distribución de las DMP en función de los sintagmas $\chi^{2}(2)=64.73, p=.000$, siendo el SP (89.14\%) el que más DMP presenta en comparación con el SV (2.17\%) y el SN (8.69\%). En el contaste entre Pr-T y la T-Ps $\chi^{2}=7.04, p=.008$, siendo el contraste Pr-T (69.56\%) en el que más DMP recae. También, en Venezuela hay diferencias entre las tónicas y las átonas $\chi^{2}=12.52, \mathrm{p}=.000$, la mayor duración con DMP recae en las tónicas (76.10\%).

\subsubsection{Oraciones declarativas}

En Colombia, respecto a la tipología acentual oracional no hay diferencias significativas $\chi^{2}(8)=4.76, p=.728$, tampoco en el contaste entre Pr-T y la T-Ps $\chi^{2}=1.92, \mathrm{p}=.166$, ni entre la tipología acentual de las palabras $\chi^{2}(2)=5.11, p=.077$, pero sí en la distribución de las DMP en función de los 
sintagmas $\chi^{2}(2)=15.38, \mathrm{p}=.000$, siendo el SV (7.70\%) el que menos DMP presenta en comparación con el SN (46.15\%) y el SP (46.15\%). Además, en Colombia la mayor duración con DMP siempre recae en la tónica.

En Venezuela, respecto a la tipología acentual oracional no hay diferencias significativas $\chi^{2}(8)=4.30, p=.829$, tampoco en el contaste entre Pr-T y la T-Ps $\chi^{2}=3.20, \mathrm{p}=.074$ ni entre la tipología acentual de las palabras $\chi^{2}(2)=3.70, p=.157$, pero sí en la distribución de las DMP en función de los sintagmas $\chi^{2}(2)=28.90, p=.000$, siendo el SP (90\%) el que más DMP presenta en comparación con el SN ( $5 \%$ ) y el SV ( $5 \%$ ). Asimismo, en Venezuela hay diferencias significativas entre la tónica y la átona $\chi^{2}=9.80$, $\mathrm{p}=.002$, siendo la tónica en la que recae la mayor frecuencia de DMP. 


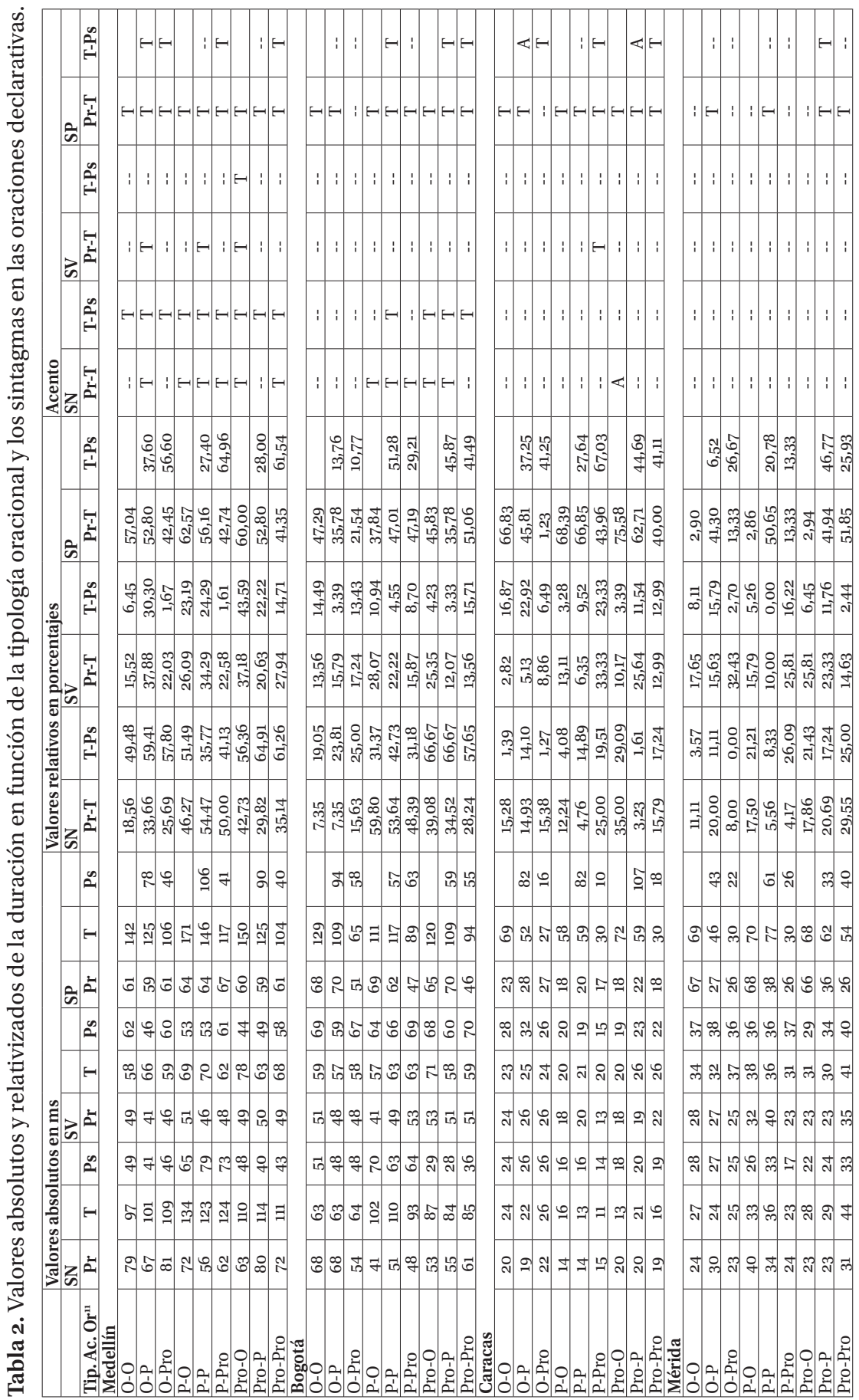

$11 \quad$ Tip. Ac. Or: Tipología Acentual Oracional. 


\subsubsection{Oraciones interrogativas}

En Colombia, respecto a la tipología acentual oracional no hay diferencias significativas $\chi^{2}(8)=5.00, p=.758$, tampoco en el contaste entre Pr-T y la T-Ps $\chi^{2}=2.50, p=.114$, pero sí en la tipología acentual de las palabras $\chi^{2}(2)=7.40, p=.025$, siendo las proparoxítonas las que presentan una distribución mayor (50\%), luego las paroxítonas (35\%) y, finalmente, las oxítonas (15\%). Asimismo, la distribución de las DMP en función de los sintagmas también presenta diferencias significativas $\chi^{2}(2)=15.05, p$ $=.001$, siendo el SP (52.50\%) el que más DMP presenta en comparación con el SN (42.50\%) y el SV (5\%). Además, en Colombia la mayor duración con DMP siempre recae en la tónica. En Venezuela, respecto a la tipología acentual oracional no hay diferencias significativas $\chi^{2}(8)=1.00$, $\mathrm{p}=.998$, tampoco en el contaste entre la tipología acentual de las palabras $\chi^{2}(2)=1.23, \mathrm{p}=.540$, pero sí entre Pr-T y la T-Ps $\chi^{2}=3.84, \mathrm{p}=.050$ siendo el contraste Pr-T (72\%) en el que se observa un mayor número de DMP. Asimismo, la distribución de las DMP en función de los sintagmas $\chi^{2}(2)=15.38, p=$.0oo, muestra que el SP (95.83\%) es en el que más DMP presenta en comparación con el SN (4.17\%), en el SV no hay DMP. Asimismo, en Venezuela también hay diferencias significativas entre la tónica y la átona $\chi^{2}=3.84, \mathrm{p}=.050$, siendo la tónica $(75 \%)$ en la que recae la mayor duración con DMP. 


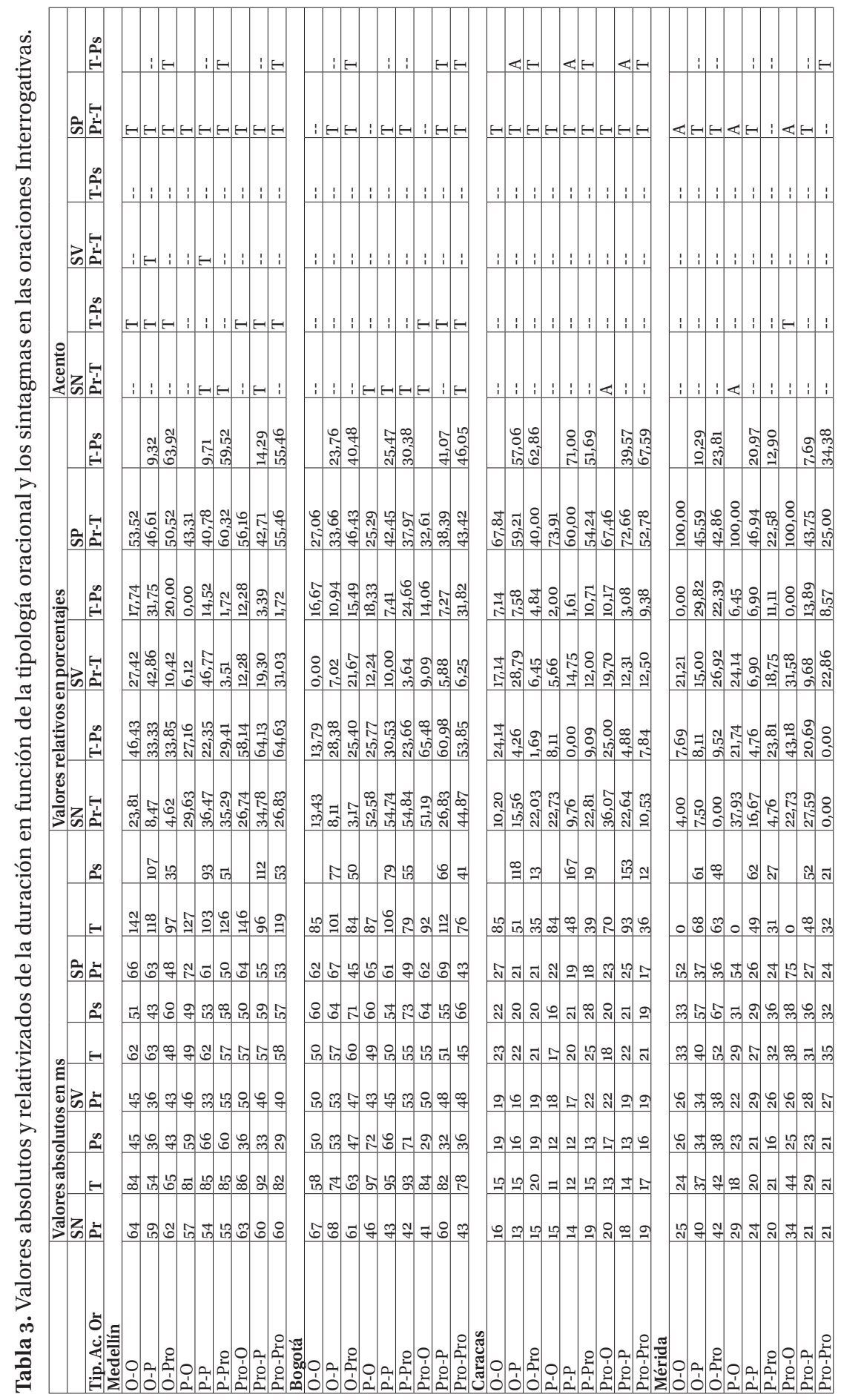




\subsection{Estructura de la duración}

La tabla 4 muestra las estructuras de la duración en la modalidad declarativa e interrogativa. En general, tomando ambos países y modalidades en conjunto los análisis señalan diferencias significativas en la distribución de las estructuras $\chi^{2}(5)=237.77, p=.000$, siendo $B^{*}(53.25 \%)$ la que presenta un mayor número, en segundo lugar $\mathrm{B}+\mathrm{L}^{*}(20.37 \%)$, a continuación $L^{*}(14.81 \%)$, seguida deL+B*(8.8\%), L+B* (2.77\%) y, finalmente, $\mathrm{B}+\mathrm{L}^{*} \mathrm{~L}$. Al comparar Colombia y Venezuela en cada una de las estructuras, los datos muestran lo siguiente: en la estructura B* Venezuela (63.48\%) presenta una mayor distribución que Colombia $(36.52 \%), \chi^{2}(1)=8.35, p=$ .004; en cambio, en la estructura $L^{*} \chi^{2}=12.50, p=.000$, Colombia (81.25\%) produce un mayor número de DMP que Venezuela (18.75\%).

Analizando ambas modalidades en conjunto, en Colombia se observa que hay diferencias significativas en la distribución $\chi^{2}(3)=15.63, p=.001$, siendo la estructura $B^{*}(38.88 \%)$ la que presenta un mayor porcentaje, en segundo lugar $B+L^{*}(25 \%)$, en tercer lugar $L^{*}(24.07 \%)$ y, finalmente, $L^{*}+B$ (12.03\%). En Venezuela, $\chi^{2}(4)=157.09, p=.000$, la estructura $B^{*}(67.62 \%)$ es la que presenta una mayor frecuencia, en segundo lugar $\mathrm{B}+\mathrm{L}^{*}(12.96 \%)$, en tercer lugar las estructuras $L^{*}$ y $L^{*}+B$ y $L+B^{*}(5.55 \%$, cada una) y, finalmente, la estructura $\mathrm{BL}^{*} \mathrm{~L}^{12}(2.77 \%)$.

12 Las estructuras $\mathrm{L}+\mathrm{B}^{*} \mathrm{y} \mathrm{BL}^{*}$ Lse registraron solamente en Venezuela. 
Tabla 4: Configuraciones de la duración en la modalidad declarativa e interrogativa en función de la tipología oracional y de los sintagmas en Colombia y Venezuela.

\begin{tabular}{|c|c|c|c|c|c|c|}
\hline \multirow{2}{*}{$\begin{array}{l}\text { Tipología acentual } \\
\text { oracional }\end{array}$} & \multicolumn{3}{|c|}{ DECLARATIVAS } & \multicolumn{3}{|c|}{ INTERROGATIVAS } \\
\hline & SN & SV & SP & SN & SV & SP \\
\hline \multicolumn{7}{|l|}{ Medellín } \\
\hline $\mathrm{O}-\mathrm{O}$ & $L * B$ & $\mathrm{~B}^{*}$ & $\mathrm{BL}^{*}$ & $\mathrm{~L} * \mathrm{~B}$ & $\mathrm{~B}^{*}$ & $\mathrm{BL}^{*}$ \\
\hline O-P & $\mathrm{L}^{*}$ & $\mathrm{BL}^{*}$ & $\mathrm{~L}^{*}$ & $\mathrm{~L}^{*} \mathrm{~B}$ & $\mathrm{BL}^{*}$ & $\mathrm{BL}^{*}$ \\
\hline O-Pro & $\mathrm{L}^{*} \mathrm{~B}$ & $\mathrm{~B}^{*}$ & $\mathrm{~L}^{*}$ & $\mathrm{~L}^{*} \mathrm{~B}$ & $\mathrm{~B}^{*}$ & $\mathrm{~L}^{*}$ \\
\hline $\mathrm{P}-\mathrm{O}$ & $\mathrm{L}^{*}$ & $\mathrm{~B}^{*}$ & $\mathrm{BL}^{*}$ & $\mathrm{~B}^{*}$ & $\mathrm{~B}^{*}$ & $\mathrm{BL}^{*}$ \\
\hline P-P & $\mathrm{L}^{*}$ & $\mathrm{BL}^{*}$ & $\mathrm{BL}^{*}$ & $\mathrm{BL}^{*}$ & $\mathrm{BL}^{*}$ & $\mathrm{BL}^{*}$ \\
\hline P-Pro & $\mathrm{L}^{*}$ & $\mathrm{~B}^{*}$ & $\mathrm{~L}^{*}$ & $\mathrm{BL}^{*}$ & $\mathrm{~B}^{*}$ & $\mathrm{~L}^{*}$ \\
\hline Pro-O & $\mathrm{L}^{*}$ & $\mathrm{~L}^{*}$ & $\mathrm{BL}^{*}$ & $\mathrm{~L}^{*} \mathrm{~B}$ & $\mathrm{~B}^{*}$ & $\mathrm{BL}^{*}$ \\
\hline Pro-P & $\mathrm{L} * \mathrm{~B}$ & $\mathrm{~B}^{*}$ & BL* & $\mathrm{L}^{*}$ & $\mathrm{~B}^{*}$ & $\mathrm{BL}^{*}$ \\
\hline Pro-Pro & $\mathrm{L}^{*}$ & $\mathrm{~B}^{*}$ & $\mathrm{~L}^{*}$ & $\mathrm{~L}^{*} \mathrm{~B}$ & $\mathrm{~B}^{*}$ & $\mathrm{~L}^{*}$ \\
\hline \multicolumn{7}{|l|}{ Bogotá } \\
\hline $\mathrm{O}-\mathrm{O}$ & $\mathrm{B}^{*}$ & $\mathrm{~B}^{*}$ & $\mathrm{BL}^{*}$ & $\mathrm{~B}^{*}$ & $\mathrm{~B}^{*}$ & $\mathrm{~B}^{*}$ \\
\hline O-P & $\mathrm{B}^{*}$ & $\mathrm{~B}^{*}$ & $\mathrm{BL}^{*}$ & $\mathrm{~B}^{*}$ & $\mathrm{~B}^{*}$ & BL* $^{*}$ \\
\hline O-Pro & $\mathrm{B}^{*}$ & $\mathrm{~B}^{*}$ & $\mathrm{~B}^{*}$ & $\mathrm{~B}^{*}$ & $\mathrm{~B}^{*}$ & $\mathrm{~L}^{*}$ \\
\hline $\mathrm{P}-\mathrm{O}$ & $\mathrm{BL}^{*}$ & $\mathrm{~B}^{*}$ & $\mathrm{BL}^{*}$ & $\mathrm{BL}^{*}$ & $\mathrm{~B}^{*}$ & $\mathrm{~B}^{*}$ \\
\hline P-P & $\mathrm{L}^{*}$ & $\mathrm{~B}^{*}$ & $\mathrm{~L}^{*}$ & $\mathrm{BL}^{*}$ & $\mathrm{~B}^{*}$ & $\mathrm{BL}^{*}$ \\
\hline P-Pro & BL* & $\mathrm{B}^{*}$ & BL* & BL* & $\mathrm{B}^{*}$ & $\mathrm{BL}^{*}$ \\
\hline Pro-O & $\mathrm{L}^{*}$ & $\mathrm{~B}^{*}$ & BL* & $\mathrm{L}^{*}$ & $\mathrm{~B}^{*}$ & $\mathrm{~B}^{*}$ \\
\hline Pro-P & $\mathrm{L}^{*}$ & $\mathrm{~B}^{*}$ & $\mathrm{~L}^{*}$ & $\mathrm{~L}^{*} \mathrm{~B}$ & $\mathrm{~B}^{*}$ & $\mathrm{~L}^{*}$ \\
\hline Pro-Pro & $\mathrm{L}^{*} \mathrm{~B}$ & $\mathrm{~B}^{*}$ & $\mathrm{~L}^{*}$ & $\mathrm{~L}^{*}$ & $\mathrm{~B}^{*}$ & $\mathrm{~L}^{*}$ \\
\hline \multicolumn{7}{|l|}{ Caracas } \\
\hline $\mathrm{O}-\mathrm{O}$ & $\mathrm{B}^{*}$ & $\mathrm{~B}^{*}$ & $\mathrm{BL}^{*}$ & $\mathrm{~B}^{*}$ & $\mathrm{~B}^{*}$ & $\mathrm{BL}^{*}$ \\
\hline $\mathrm{O}-\mathrm{P}$ & $\mathrm{B}^{*}$ & $\mathrm{~B}^{*}$ & BL*L & $\mathrm{B}^{*}$ & $\mathrm{~B}^{*}$ & $B L^{*} \mathrm{~L}$ \\
\hline O-Pro & $\mathrm{B}^{*}$ & $\mathrm{~B}^{*}$ & $\mathrm{~L}^{*} \mathrm{~B}$ & $\mathrm{~B}^{*}$ & $\mathrm{~B}^{*}$ & $\mathrm{~L}^{*}$ \\
\hline $\mathrm{P}-\mathrm{O}$ & $\mathrm{B}^{*}$ & $\mathrm{~B}^{*}$ & $\mathrm{BL}^{*}$ & $\mathrm{~B}^{*}$ & $\mathrm{~B}^{*}$ & $\mathrm{BL}^{*}$ \\
\hline P-P & $\mathrm{B}^{*}$ & $\mathrm{~B}^{*}$ & $\mathrm{BL}^{*}$ & $\mathrm{~B}^{*}$ & $\mathrm{~B}^{*}$ & BL*L \\
\hline P-Pro & $\mathrm{B}^{*}$ & $\mathrm{BL}^{*}$ & $\mathrm{~L}^{*}$ & $\mathrm{~B}^{*}$ & $\mathrm{~B}^{*}$ & $\mathrm{~L}^{*}$ \\
\hline Pro-O & LB $^{*}$ & $\mathrm{~B}^{*}$ & $\mathrm{BL}^{*}$ & $\mathrm{LB}^{*}$ & $\mathrm{~B}^{*}$ & $\mathrm{BL}^{*}$ \\
\hline Pro-P & $\mathrm{B}^{*}$ & $\mathrm{~B}^{*}$ & BL* ${ }^{*}$ & $\mathrm{~B}^{*}$ & $\mathrm{~B}^{*}$ & $\mathrm{BL}^{*} \mathrm{~L}$ \\
\hline Pro-Pro & $\mathrm{B}^{*}$ & $\mathrm{~B}^{*}$ & $\mathrm{~L}^{*}$ & $\mathrm{~B}^{*}$ & $\mathrm{~B}^{*}$ & $\mathrm{~L}^{*}$ \\
\hline \multicolumn{7}{|l|}{ Mérida } \\
\hline $\mathrm{O}-\mathrm{O}$ & $\mathrm{B}^{*}$ & $\mathrm{~B}^{*}$ & & $\mathrm{~B}^{*}$ & $\mathrm{~B}^{*}$ & LB* $^{*}$ \\
\hline O-P & $\mathrm{B}^{*}$ & $\mathrm{~B}^{*}$ & $\mathrm{BL}^{*}$ & $\mathrm{~B}^{*}$ & $\mathrm{~B}^{*}$ & $\mathrm{BL}^{*}$ \\
\hline O-Pro & $\mathrm{B}^{*}$ & $\mathrm{~B}^{*}$ & $\mathrm{~B}^{*}$ & $\mathrm{~B}^{*}$ & $\mathrm{~B}^{*}$ & $\mathrm{BL}^{*}$ \\
\hline $\mathrm{P}-\mathrm{O}$ & $\mathrm{B}^{*}$ & $\mathrm{~B}^{*}$ & $\mathrm{~B}^{*}$ & $\mathrm{LB}^{*}$ & $\mathrm{~B}^{*}$ & LB* $^{*}$ \\
\hline P-P & $\mathrm{B}^{*}$ & $\mathrm{~B}^{*}$ & $\mathrm{BL}^{*}$ & $\mathrm{~B}^{*}$ & $\mathrm{~B}^{*}$ & BL* \\
\hline P-Pro & $\mathrm{B}^{*}$ & $\mathrm{~B}^{*}$ & $\mathrm{~B}^{*}$ & $\mathrm{~B}^{*}$ & $\mathrm{~B}^{*}$ & $\mathrm{~B}^{*}$ \\
\hline Pro-O & $\mathrm{B}^{*}$ & $\mathrm{~B}^{*}$ & $\mathrm{~B}^{*}$ & $\mathrm{~L}^{*} \mathrm{~B}$ & $\mathrm{~B}^{*}$ & LB* $^{*}$ \\
\hline Pro-P & $\mathrm{B}^{*}$ & $\mathrm{~B}^{*}$ & $L^{*}$ & $\mathrm{~B}^{*}$ & $\mathrm{~B}^{*}$ & $\mathrm{BL}^{*}$ \\
\hline Pro-Pro & $\mathrm{B}^{*}$ & $\mathrm{~B}^{*}$ & $\mathrm{BL}^{*}$ & $\mathrm{~B}^{*}$ & $\mathrm{~B}^{*}$ & $\mathrm{~L}^{*} \mathrm{~B}$ \\
\hline
\end{tabular}


Considerando cada modalidad por separado, en las oraciones declarativas de Colombia la estructura que presenta un mayor porcentaje de ocurrencia en el SN es L*, en el SV es B* y en el SP, con un porcentaje similar, las estructuras $B+L^{*}$ y L*. En Venezuela, por su parte, la estructura que tieneun mayor porcentaje de ocurrencia en losSN y SV es B*, mientras que en el SP es B+L*. En lo que respecta a las oraciones interrogativas, en Colombia, la estructura que presenta un mayor porcentaje de ocurrencia en el SN es $\mathrm{L}^{*}+\mathrm{B}$; en el SV es B* y en el SP es B+L*. En Venezuela, la estructura que tiene un mayor porcentaje de ocurrencia en los SN y SV es $\mathrm{B}^{*}$ mientras que en el SP es $\mathrm{B}+\mathrm{L}^{*}$ (Ver tabla 5 ).

Tabla 5: Porcentaje de la estructuras de la duración en la modalidad declarativa e interrogativa en Colombia y Venezuela ${ }^{13}$.

\begin{tabular}{|c|c|c|c|c|c|c|c|c|c|c|c|c|}
\hline & \multicolumn{2}{|c|}{ SN } & \multicolumn{2}{|c|}{ SV } & \multicolumn{2}{|r|}{ SP } & \multicolumn{2}{|c|}{ SN } & \multicolumn{2}{|c|}{ SV } & \multicolumn{2}{|c|}{ SP } \\
\hline \multicolumn{7}{|c|}{ Colombia } & \multicolumn{6}{|c|}{ Venezuela } \\
\hline & $\%$ & Estr. & $\%$ & Estr. & $\%$ & Estr. & $\%$ & Estr. & $\%$ & Estr. & $\%$ & Estr. \\
\hline \multirow{4}{*}{ I } & 16.7 & $\mathrm{~L}^{*}$ & 27.8 & $\mathrm{~B}^{*}$ & 13.0 & $\mathrm{BL}^{*} ; \mathrm{L}^{*}$ & 31.5 & $\mathrm{~B}^{*}$ & 31.5 & $\mathrm{~B}^{*}$ & 11.1 & $\mathrm{BL}^{*}$ \\
\hline & 7.4 & $\mathrm{~L}^{*} \mathrm{~B}$ & 3.7 & $\mathrm{BL}^{*}$ & 5.6 & $\mathrm{~L}^{*} \mathrm{~B}$ & 1.9 & $\mathrm{LB}^{*}$ & 1.9 & $\mathrm{BL}^{*}$ & 5.6 & $\mathrm{~L}^{*}$ \\
\hline & 5.6 & $\mathrm{~B}^{*}$ & 1.9 & $\mathrm{~L}^{*}$ & 1.9 & $\mathrm{~B}^{*}$ & & & & & 9.3 & $\mathrm{~B}^{*}$ \\
\hline & 3.7 & $\mathrm{BL}^{*}$ & & & & & & & & & 7.4 & $\mathrm{~L}^{*} \mathrm{~B}$ \\
\hline \multirow{4}{*}{ I } & 11.1 & $\mathrm{~L}^{*} \mathrm{~B}$ & 29.6 & $\mathrm{~B}^{*}$ & 16.7 & $\mathrm{BL}^{*}$ & 27.8 & $\mathrm{~B}^{*}$ & 33.3 & $\mathrm{~B}^{*}$ & 13.0 & $\mathrm{BL}^{*}$ \\
\hline & 9.3 & $\mathrm{BL}^{*}$ & 3.7 & $\mathrm{BL}^{*}$ & 11.1 & $\mathrm{~L}^{*}$ & 3.7 & $\mathrm{LB}^{*}$ & & & 5.6 & $\begin{array}{l}\mathrm{L}^{*} \mathrm{~B} \\
\mathrm{~L}^{*} ; \\
\mathrm{BL}^{*} \mathrm{~L}\end{array}$ \\
\hline & 7.4 & $\mathrm{~B}^{*}$ & & & 5.6 & $\mathrm{~B}^{*}$ & 1.9 & $\mathrm{~L}^{*} \mathrm{~B}$ & & & 1.9 & $\mathrm{~B}^{*} ; \mathrm{L}^{*}$ \\
\hline & 5.6 & $\mathrm{~L}^{*}$ & & & & & & & & & & \\
\hline
\end{tabular}

\section{Discusión y conclusiones}

El objetivo principal de este trabajo fue comparar la duración del habla de informantes femeninas pertenecientes a dos de las ciudades más importantes de dos países fronterizos: Bogotá y Medellín (en Colombia) y Caracas y Mérida (en Venezuela). Para ello, se observó, en primer lugar, el comportamiento de la duración basado en el análisis de las medias generales; en segundo, la distribución de las DMP y, finalmente, se generó la estructura de la duración basada en la propuesta de vocales largas y breves.

13 Las abreviaturas de la tabla son: Estr.: estructura; SV: sintagma verbal; Pr: pretónica, T: tónica, Ps: Postónica, O: oxítona, P: paroxítona y Pr: proparoxítona; D: declarativa; I: interrogativa. 
El análisis de las medias generales mostró que la duración de las vocales del habla colombiana es mayor que en Venezuela independientemente de la modalidad y de la tipología acentual. Sin embargo, en ambos paísesy en las dos modalidades, la duración de las vocales del SP es sensiblemente mayor que las del SN. Además, en el SP las oxítonas son las que presentan una mayor duración y las proparoxítonas las que tienen menor duración: se exceptúa el SP de las interrogativas de Venezuela en donde las paroxítonas tienen una mayor duración. Por tanto, las medias generales de cada país muestran que la duración de las vocales tónicas tiende a ser mayor a medida que se aproximan al límite sintagmático. Los resultados están en línea con planteamientos actuales que sugieren que hay una mayor duración de las últimas sílabas en la frase, como, por ejemplo, aquellos reportados para hablantes canarios y cubanos (Dorta, Hernández, y Martín, 2013), suizos (Lindblom, 1968), catalanes (Fernández Planas y Martínez Celdrán, 2003; Fernández Planas y otros, 2007; Szmidt y Labraña, 2013), vascos (Elejabeitia, Iribar, y Pagola, 2005) entre otros.

Respecto al análisis de la distribución de las DMP, Colombia presenta un porcentaje mayor de contrastes que Venezuela, siendo Medellín la que tiene una mayor frecuencia y Mérida la que menos. El análisis de la tipología oracional no arroja datos significativos ni por países ni por modalidades lo cual implica que el porcentaje de DMP es similar en las oraciones con independencia de dichas variables. En términos generales, la vocal tónica muestra mayor duración que la pretónica y la postónica en la totalidad de los casos en Colombia; sin embargo, en Venezuela, aunque raramente, en algunas ocasiones la pretónica o la postónica es más larga que la tónica. En relación con el análisis de los sintagmas, en Colombia suele haber un porcentaje similar de DMP en el SN y en el SP en ambas modalidades oracionales, siendo este porcentaje significativamente mayor al del SV. También, hay un porcentaje similar en los contrastes entre Pr-T y la T-Ps. En Venezuela, suele haber un porcentaje similar entre el SN y el SV en las declarativas, pero en las interrogativas no hay DMP en el SV. El porcentaje de estos sintagmas es significativamente inferior al SP en ambas modalidades oracionales. Además, en las oraciones interrogativas venezolanas, estas diferencias se encuentran principalmente en el contraste Pr-T.

En cuanto a la tipología acentual, solamente se encuentran DMP en la modalidad interrogativa del habla colombiana. La mayor distribución se 
presenta en las proparoxítonas, luego en las paroxítonas y finalmente en las oxítonas. Así, teniendo en cuenta los resultados relacionados con las medias, aunque las oxítonas tienen mayor duración, las proparoxítonas tienden a presentan mayor porcentaje de DMP respecto a la pretónica y postónica en las interrogativas del habla colombiana.

Respecto a la propuesta de la estructura de la duración en general, la que presenta mayor frecuencia en ambos países es, en primer lugar, $B^{* 14}$, en segundo lugarB $+L^{*}$, en tercer lugar $L^{*} y$, finalmente, $L^{*}+B$ aunque en Venezuela, a diferencia de Colombia, las dos últimas estructuras se presentan con un porcentaje similar. Además, en Venezuela la estructura $\mathrm{L}+\mathrm{B}^{*}$ tiene la misma frecuencia que las dos últimas estructuras mencionadas.

Específicamente, la estructura que subyace en el habla colombina en las oraciones declarativas es: en el SN, L* y en el SV, B*; en el SP, en cambio, alternan dos estructuras: $\mathrm{B}+\mathrm{L}^{*} \mathrm{y} \mathrm{L}^{*}$. En la modalidad interrogativa: en el SN, $L^{*}+B$; en el SV, B* y en el SP, B+L*. En Venezuela, en la oración declarativa primaría: en el SN, $B^{*}$, en el SV, B* y en el SP, B+L*. En las interrogativas, en el SN, B*; en el SV, B* y en el SP, B+L*. Así, en Colombia, la diferencia entre las modalidades estaría básicamente en el SN, pues en el SP ambas modalidades coinciden en la misma estructura. En cambio, en Venezuela ambas modalidades coinciden en las estructuras más frecuentes en los tres sintagmas. Los análisis muestran que la estructura $B^{*}$ se ubica con mayor frecuencia en el SV en ambos países y en el SN de la voz venezolana. No obstante, en el SP, independientemente del país o de la modalidad, el mayor porcentaje lo obtiene la estructura $\mathrm{B}+\mathrm{L}^{*} \mathrm{o}$ $\mathrm{L}^{*}$ (solamente en Colombia) cuya característica fundamental es que la tónica es significativamente más larga que la pretónica o que la postónica. Este resultado, unido al ya mencionado respecto a que las tónicas del SP en relación a las tónicas del SN y del SV son más largas, apoya la idea de que la duración es un parámetro determinante para marcar el tonema, como lo sugiere Fernández Planas y Martínez Celdrán (2003). En términos generales, Colombia presenta un porcentaje mayor que Venezuela en la estructura L*. Asimismo, en el habla colombiana esta estructura obtiene el máximo porcentaje en el SN de las oraciones declarativas, en las interrogativas no se presenta; y también obtiene un alto porcentaje

14 Los datos previos muestran que $B^{*}$ es significativamente más frecuente en Venezuela que en Colombia. 
en el SP de ambas modalidades. En Venezuela, aunque la estructura se produce, representa el segundo porcentaje más alto del SP.

\section{Conclusión general}

La duración del español hablado en Colombia difiere de la de Venezuela porque las vocales colombianas suelen ser más largas; las tónicas son siempre las que tienen mayor duración; las interrogativas muestran diferencias entre la tipología acentual siendo las proparoxítonas las que presentan un número mayor de DMP; la estructura de la duración es diferente entre las modalidades. Finalmente, en Colombia el SN y el SP presentan DMP con una frecuencia similar; en Venezuela la mayor frecuencia está en el SP. Sería interesante profundizar en estos estudios con el fin de generar conclusiones con un espectro más amplio.

\section{Referencias bibliográficas}

Alfano, I., Llisterri, J., y Savy, R. (2007). The perception of Italian and Spanish lexical stress: A first cross-linguistic study. In ICPhS 2007. Proceedings of the $16^{\text {th }}$ International Congress of Phonetic Sciences. [CD-ROM] (pp. 1793-6). Saarbrücken, Germany, 6-10 August. 2007. Documento de internet disponibleenhttp://liceu. uab.cat/ joaquim/publicacions/Alfano_Llisterri_Savy_07_ Perception_Stress_Spanish_Italian.pdf

Almeida, M. (1999). Tiempo y ritmo en el español canario. Un estudio acústico. Madrid: Iberoamericana Vervuert.

Bello, A. (1955). Principios de la ortología y métrica de la lengua castellana y otros escritos. Caracas: Ed. del Ministerio de Educación.

Canellada, M. y Madsen, J. (1987). Pronunciación del español. Lengua hablada y literaria. Madrid: Castalia.

Cutler, A., Dahan, D. y Van Donselaar, W. (1997). Prosody in the comprehension of spoken language: a literature review. Language and speech, 40(2), 141-201.

Cyrille, M., Astésano, C., Aramaki, M., Ystad, S., Kronland, R. y Besson, M. (2007). Influence of Syllabic Lengthening on Semantic Processing in Spoken French: Behavioral and Electrophysiological Evidence. Cerebral Cortex, (17), 2659-2668. 
Díaz, Ch., Muñetón, M. y Dorta, J. (2017). Estudio comparativo de la entonación en habla formal femenina de Caracas (Venezuela) y Bogotá (Colombia). Revista Internacional de Lingüística Iberoamericana (RILI), 15(1,29), 237-256.

Díaz, Ch., Dorta, J., Mora, E. y Muñetón, M. (en prensa). Intonation across Two Border Areas in the North Andean Region. Mérida Venezuela and Medellin Colombia. En John Benjamins (Eds.), Spanish in Context.

Dorta, J., Hernández Díaz, B., y Díaz, Ch. (2009). Interrogativas absolutas: relación entre F0, duración e intensidad. Estudios de Fonética Experimental, (18), 123-144.

(2011). Duración e intensidad en la entonación de las declarativas e interrogativas de Canarias. En F. Hernández González, M. Martínez Hernández y L. M. Pino Campos (Eds.), Sosalivm Mvnera, Homenaje a Francisco González Luis (pp. 143-154). Madrid: Ediciones Clásica.

Dorta, J. y Mora, E. (2011). Patrones temporales en dos variedades del español hablado en Venezuela y Canarias. Revista Internacional de Lingüística Iberoamericana (RILI). Monográfico: La prosodia en lenguas y variedades del ámbito iberorrománico, (9), 91-100.

Dorta, J., Hernández, B. y Martín, J. (2013). Comparación de la entonación canario cubana. Josefa Dorta (Ed.), Estudio comparativo preliminar de la entonación de Canarias, Cuba y Venezuela (pp. 87-168). Madrid-Santa Cruz de Tenerife: La Página ediciones S/L, Colección Universidad.

Dorta, J. y Jorge Trujillo, C. (2015). Estudios prosódicos en Canarias: análisis de la duración en habla formal de El Hierro y Fuerteventura. Estudios de Fonética Experimental, (24), 11-33.

Elejabeitia, A., Iribar, A. y Pagola, R. M. (2005).Notas sobre la prosodia del castellano de Bizkaia. Estudios de Fonética Experimental, (14), 247-272.

Escandell-Vidal, V. (2011). Verumfocus y prosodia: Cuando la duración (sí que) importa. Oralia, (14), 181-201.

Fernández Planas, A., Martínez Celdrán, E., Van Oosterzee, C., SalcioliGuidi, V., Castellví Vives, J. y Szmidt, D. (2007). Proyecto AMPER: estudio contrastivo de frases interrogativas sin expansión del barceloníy del tarragoní. En Cano López y 
otros (Eds.), Actas del VI Congreso de Lingüística General (pp. 1931-1943), Madrid: Arco/libros.

Fernández Planas, A. y Martínez Celdrán, E. (2003). El tono fundamental y la duración: dos aspectos de la taxonomía prosódica en dos modalidades de habla (enunciativa e interrogativa) del español. Estudios de Fonética Experimental, 12, 166-200.

Fox, A. (2000). Prosodic features and prosodic structure. Oxford, NY: Oxford University Press.

Garrido, J., Llisterri, J., De la Mota, C. y Ríos, A. (1995).Estudio comparado de las características prosódicas de la oración simple en español en dos modalidades de lectura. En Elejabetia, A. y Iribar, A. (Eds.), Phonetica. Trabajos de fonética experimental (pp.173194). Bilbao: Universidad de Deusto (Serie Lingüística, 6).

Lindblom, B. (1968). Temporal organization of syllable production. SpeechTransmission Laboratory Quarterly Progress status Report, 3(2), 1- 5.

Llisterri, J., Machuca, M. J., de la Mota, C., Riera, M., y Ríos, A. (2005). La percepción del acento léxico en español. En Filología y lingüistica. Estudios ofrecidos a Antonio Quilis (pp. 271-97). Madrid: Consejo Superior de Investigaciones Científicas -Universidad Nacional de Educación a Distancia- Universidad de Valladolid.

López Bobo, M., Muñiz, C., Díaz, L., Corral, N., Brezmes, D. y Alvarellos, M. (2007). Análisis y representación de la entonación. Replanteamiento metodológico en el marco del proyecto AMPER. En Dorta, Josefa (Ed.), La prosodia en el ámbito lingüístico románico (pp. 17-34). Madrid/Tenerife: La Página ediciones S. L. /Colección Universidad.

Marrero, V. (2008). La fonética Perceptiva: trascendencia lingüística de mecanismos neurofisiológicos. Estudios de Fonética Experimental, (17), 207-245.

Mora, E. y Asuaje, R. (2009). El canto de la palabra: una iniciación al estudio de la prosodia. Mérida: Universidad de Los Andes.

Mora, E., Villamizar, T., Blondet, M. y López, Y. (1999). Hacia una caracterización rítmica del español hablado en Venezuela. Boletín Antropológico, (47), 75-87. 
Muñetón, M. (2016). La F0, duración e intensidad de las oraciones interrogativas absolutas en un informante varón de Medellín. Estudios de Fonética Experimental, (25), 167-192.

Pamies Bertrán, A., y Fernández Planas, A. (2006). La percepción de la duración vocálica en español. En J. D. Luque Durán (Ed.), Actas del V Congreso Andaluz de Lingüistica General. Homenaje al Profesor José Andrés de Molina Redondo I (pp. 501-512). Granada: Lingvistica-Ediciones Método.

Salverda, A., Dahan, D. y McQueen, J. (2003). The role of the prosodic boundaries in the resolution of lexical embedding in speech comprehension. Cognition, (90), 51 - 89.

Szmidt, D. y Labraña, S. (2013). Entonación de las oraciones interrogativas absolutas neutras en el catalán mallorquín. Comparación con el catalán central de Barcelona. Onomázein, (27), 286-297.

Toledo, G. (2000). Taxonomía tonal en español. LanguageDesign, (3), $1-20$. 